\begin{tabular}{|c|c|}
\hline Title & Weakly nonlinear waves radiated by pulsations of a cylinder \\
\hline Author(s) & Y ano, Takeru; Inoue, Y oshinori \\
\hline Citation & $\begin{array}{l}\text { The Journal of the A coustical Society of A merica, 93(1), 132-141 } \\
\text { https://doi.org/10.1121/1.405650 }\end{array}$ \\
\hline Issue Date & 1993-01 \\
\hline Doc URL & http:/hdl.handle.net/2115/44915 \\
\hline Rights & $\begin{array}{l}\text { Copyright 1993, A coustical Society of A merica. This article may be downloaded for personal use only. A ny other use } \\
\text { requires prior permission of the author and the A coustical Society of A merica. }\end{array}$ \\
\hline Type & article \\
\hline File Information & JA SA 93 1.pdf \\
\hline
\end{tabular}

Instructions for use 


\title{
Weakly nonlinear waves radiated by pulsations of a cylinder
}

\author{
Takeru Yano and Yoshinori Inoue \\ Department of Engineering Science, Faculty of Engineering, Hokkaido University. Sapporo 060, Japan
}

(Received 24 March 1992; accepted for publication 25 August 1992)

\begin{abstract}
A cylindrical sound wave is radiated into an unbounded ideal gas by an infinite circular cylinder that executes a sinusoidal pulsation uniformly along its axis with small amplitude and moderate frequency for only one period. In the leading order of approximation, the weakly nonlinear propagation of the wave is studied for the case of sufficiently large acoustic Reynolds number up to the stage that its profile develops into a cylindrical $N$ wave with a tail. In the near field, solving the linear wave equation gives the near-field solution, which has a tail following the body part of the wave. The tail of the velocity profile decreases asymptotically in proportion to $t^{*-4}$ as $t^{*} \rightarrow \infty$ at a fixed point in the near field and to $t^{*-5 / 2}$ at a fixed point in the far field ( $t *$ is the time from the beginning of the pulsation). The tail vanishes in a high frequency limit with an acoustic Mach number being fixed. In the far field, an exact solution, which matches with the near-field solution, is obtained for a far-field equation by the method of strained coordinates. The evolution of the two shocks and the tail are then examined by making use of the equal-areas rule. At large distances from the cylinder, the whole profile of the wave approaches to an $N$ wave with a long tail.
\end{abstract}

PACS numbers: 43.25.Cb, 43.28.Mv, 43.30.Qd

\section{INTRODUCTION}

The weakly nonlinear propagation of cylindrical waves has been studied as a problem of fundamental importance in nonlinear acoustics. ${ }^{1-4}$ We shall consider a nonperiodic problem, where an infinite circular cylinder in an unbounded ideal gas executes a sinusoidal pulsation uniformly along its axis for only one period. The radiated sound wave consists of a sinusoidal body part and a tail. At large distances from the cylinder, the whole profile of the wave approaches to a cylindrical $N$ wave with a tail. An analogous nonperiodic problem has recently been studied for a spherical wave by Inoue et al., ${ }^{5}$ where the wave is radiated by a sphere's sinusoidal pulsation for one period; the asymptotic profile is a spherical $N$ wave with a tail. In what follows we shall extend the analysis in Ref. 5 to the cylindrical wave with a tail.

The propagation of the cylindrical $N$ wave has been well examined, ${ }^{3.6,7}$ so that its decay rate and an asymptote of the shock path are well known for an ordinary balanced $N$ wave with no tail (e.g., Landau and Lifshitz, ${ }^{6}$ Whitham ${ }^{7}$ ). On the other hand, in spite of the fact that a cylindrical sound wave radiated by a source of finite duration can have a tail, ${ }^{8,9}$ the detailed picture has not been clarified for the process of transformation of the radiated sound wave with a tail into an $N$ wave with a tail. We shall here investigate the propagation of the weakly nonlinear cylindrical wave in the leading order of approximation up to the stage that the wave profile develops into an $N$ wave with a tail. The behavior of the tail is also examined in detail. The resulting wave phenomena are as a whole similar to those for the spherical wave, ${ }^{5}$ with the exception of the behavior of the tail. The solution is represented by using not only the Bessel functions but also an integral of the modified Bessel functions, while for the spherical wave in Ref. 5 the solution can be expressed in terms of the exponential and trigonometric functions.
The problem considered here pertains to the case of $\epsilon \equiv a / \bar{R} \ll 1$ and $\Omega \equiv \bar{R} \omega / c=O(1)$ (small amplitude and moderate frequency case ), ${ }^{10}$ where $\epsilon$ is a normalized amplitude and $\Omega$ is a normalized angular frequency ${ }^{11}(\bar{R}$ : the mean radius of the cylinder; $a$ : an amplitude of the sinusoidal pulsation of the cylinder; $\omega$ : an angular frequency of the pulsation; $c$ : the speed of sound in an initial undisturbed gas). The radiated sound field is then composed of the near and far field. The condition $\Omega=O(1)$ implies that the near field has the dimensions comparable with $\bar{R}$, in which the linear theory in acoustics is valid in the leading order of approximation. An acoustic Mach number $M$ is then sufficiently small:

$$
M \equiv a \omega / c=\epsilon \Omega \ll 1,
$$

which means that the nonlinearity is weak. We also suppose that an acoustic Reynolds number $R_{c}$ is sufficiently large:

$$
R_{c} \equiv c a / \delta^{*} \gg 1,
$$

where $\delta^{*}$ is the diffusivity of sound given as $\delta^{*}=\left[\left(4 \eta^{*} / 3\right)+\zeta^{*}+(\gamma-1) \kappa / c_{p}\right] / \rho_{0} \quad\left(\eta^{*}:\right.$ the shear viscosity; $\zeta^{*}$ : the bulk viscosity; $\kappa$ : the thermal conductivity; $\gamma$ : the ratio of specific heats; $c_{p}$ : the specific heat at constant pressure; $\rho_{0}$ : the density in an initial undisturbed gas). ${ }^{12}$ Condition (2) allows neglecting the dissipation effect at least until a shock wave is formed. Once a shock is formed, we can regard it as a discontinuity under this condition. The equal-areas rule is accordingly available for analyzing the evolution of the shock. ${ }^{6.7}$ In a great distant region, the cylindrical shocks concerned may no longer be thin compared with the overall scale of the $N$ wave ${ }^{13}$ Such an "old age" behavior is not discussed in this paper.

In the high frequency limit of $\Omega \rightarrow \infty$ with $M=\epsilon \Omega$ fixed, the near field substantially disappears and the overall acoustic field may be regarded as the far field. This limiting 
case is, however, essentially embraced within the present case of $\epsilon \ll 1$ and $\Omega=O(1)$ if we restrict ourselves to the lowest-order problem. ${ }^{4,14}$ In this limit, the tail vanishes, as in the spherical wave. ${ }^{5}$ The problem treated by Sachdev and Seebass $^{3}$ pertains to the case of large $\Omega=O\left(M^{-1}\right)(M \ll 1)$ and hence no tail has appeared. We shall examine the behavior of the tail in detail: in the velocity profile, it decreases asymptotically in proportion to $t^{*-4}$ as $t^{*} \rightarrow \infty$ at a fixed point in the near field and to $t^{*-5 / 2}$ at a fixed point in the far field $\left(t^{*}\right.$ : the time from the beginning of the source motion ), whereas, according to Ref. 5 , the tail of the spherical wave decays as $e^{-t^{*}}$ at a fixed point in both the near and far field.

The problem considered in this paper is relevant to that of a steady supersonic flow past a pointed slender body of revolution with finite length: the wave pattern received at large distances from the body is an $N$ wave with a tail (sonic boom). Whitham ${ }^{15}$ has studied this problem; his result for the behavior of the tail far from the body is very similar to that in the present problem. The problem of the supersonic flow however corresponds to that of pulsating cylinder in the case of $\epsilon=O(1)$ and $\Omega \ll 1$ (large amplitude and low frequency case $)^{10}$ rather than the present case, because fluid particles on the body "oscillate" between the pointed noses of the body along its surface (this means "large amplitude") and because the maximum radius of the body is very small compared with a typical wavelength (this means "low frequency"). Although here we are not concerned with the large amplitude and low frequency case, the result by Inoue and Gotoh ${ }^{16}$ may be worth noting: They have considered weakly nonlinear propagation of spherical waves radiated by a sphere executing a steady sinusoidal pulsation in the case of $\epsilon=O(1)$ and $\Omega \ll 1$. According to the result, two shocks can be formed in each one wavelength, ${ }^{17}$ while, in the small amplitude case, only one shock is formed in one wavelength. ${ }^{18}$ They have also shown that the shock formation distance is very large and hence the shock strength is very weak, compared with those in the case of $\epsilon \ll 1$ and $\Omega=O(1)$ for a given $M=\epsilon \Omega$.

In Sec. I we shall formulate the problem mathematically. The linear problem in the near field is solved in Sec. II. The near-field solution, i.e., the exact solution of the linear wave equation satisfying the boundary condition on the mean surface of the cylinder, is given so as to describe the three regions explicitly: region (I) is a constant state, and regions (II) and (III) correspond to a body part and a tail, respectively. The nonlinear propagation in the far field is examined in Sec. III. We shall apply the method of strained coordinates to obtain an explicit representation of an exact solution of a far-field equation. The shock formation distances for two shocks are estimated from the far-field solution. The evolution of the $N$ wave with the tail is analyzed with the help of the equal-areas rule in Sec. IV.

\section{FORMULATION OF THE PROBLEM}

We shall consider the weakly nonlinear propagation of a cylindrical wave radiated in an unbounded ideal gas by a pulsation of an infinite circular cylinder. The cylinder is supposed to execute a sinusoidal pulsation which lasts for only one period, i.e., $0<t^{*}<2 \pi / \omega$, where $t^{*}$ is the time and $\omega$ is an angular frequency of the pulsation. The pulsation of the cylinder is also assumed to be uniform along its axis, in the case where there is no variation in the acoustic response in the axial direction. The particle velocity of the fluid has thus only the radial component $u^{*}\left(r^{*}, t *\right)$, where $r^{*}$ is the distance from the axis of the cylinder. The wave motion concerned can be described in terms of a velocity potential $\Phi^{*}\left(r^{*}, t^{*}\right)$ such that $u^{*}=\partial \Phi^{*} / \partial r^{*}$. The following dimensionless quantities are introduced:

$\Phi=\frac{\Phi^{*}}{c \bar{R}}, \quad t=\frac{t^{*} c}{\bar{R}}, \quad r=\frac{r^{*}}{\bar{R}}, \quad u=\frac{u^{*}}{c}, \quad p=\frac{p^{*}}{p_{0}}-1$,

where $c$ is the speed of sound in an initial undisturbed gas, $\bar{R}$ is the mean radius of the cylinder, $p^{*}$ is the pressure, and $p_{0}$ is the initial undisturbed pressure.

We shall assume that the acoustic Reynolds number $R_{c}$ is sufficiently large compared with unity [see Eq. (2)]. The dissipation effect can therefore be ignored at least until a shock wave is formed. The equation governing the wave motion is then written as ${ }^{18}$

$$
\begin{aligned}
\Delta \Phi-\frac{\partial^{2} \Phi}{\partial t^{2}}= & \frac{\partial}{\partial t}\left[\left(\frac{\partial \Phi}{\partial r}\right)^{2}+\frac{\gamma-1}{2}\left(\frac{\partial \Phi}{\partial t}\right)^{2}\right] \\
& +(\text { cubic terms in } \Phi),
\end{aligned}
$$

where $\gamma$ is the ratio of specific heats ${ }^{19}$ and $\Delta$ is the Laplacian operator defined as

$$
\Delta \equiv \frac{\partial^{2}}{\partial r^{2}}+\frac{1}{r} \frac{\partial}{\partial r} .
$$

The normalized radial velocity $u$ and the normalized pressure disturbance $p$ can be expressed in terms of the nondimensional velocity potential $\Phi$ as

$$
\begin{aligned}
& u=\frac{\partial \Phi}{\partial r}, \\
& (1+p)^{(\gamma-1) / \gamma}=1-(\gamma-1)\left[\frac{\partial \Phi}{\partial t}+\frac{1}{2}\left(\frac{\partial \Phi}{\partial r}\right)^{2}\right] .
\end{aligned}
$$

The gas is assumed to be uniform and at rest for $t \leqslant 0$. The initial conditions for $\Phi$ are therefore

$$
\Phi=\frac{\partial \Phi}{\partial t}=0 \quad \text { at } t=0 .
$$

The boundary condition on the surface of the cylinder is

$$
\frac{\partial \Phi}{\partial r}=\frac{d R(t)}{d t} \text { at } r=R(t) .
$$

Here, $R(t)$, the instantaneous radius of the cylinder normalized by $\bar{R}$, is given as

$$
R(t)= \begin{cases}1-\epsilon \cos \Omega t, & \text { for } 0 \leqslant t<T, \\ 1-\epsilon, & \text { for } t<0 \text { and } T \leqslant t,\end{cases}
$$

where $\epsilon \equiv a / \bar{R}(\ll 1)$ is the normalized amplitude of the pulsation, $\Omega \equiv \bar{R} \omega / c[=O(1)]$ is the normalized angular frequency, and $T \equiv 2 \pi / \Omega$ is a normalized period (or a normalized wavelength). Since we are concerned with a weakly nonlinear problem, the acoustic Mach number $M=\epsilon \Omega$ should be sufficiently small compared with unity [see Eq. 
(1) ]. The radiation condition should be imposed on the velocity potential at infinity.

\section{LINEAR PROPAGATION IN THE NEAR FIELD}

Firstly, we shall determine the acoustic behavior in the lowest order for the near field. Neglecting the nonlinear terms in Eq. (4) leads to the linear wave equation:

$$
\frac{\partial^{2} \Phi}{\partial r^{2}}+\frac{1}{r} \frac{\partial \Phi}{\partial r}-\frac{\partial^{2} \Phi}{\partial t^{2}}=0 .
$$

The boundary condition (9) with (10) can be reduced to

$$
\frac{\partial \Phi}{\partial r}=\epsilon \Omega \sin \Omega t[H(t)-H(t-T)] \text { at } r=1,
$$

where $H(t)$ is the Heaviside unit step function defined by

$$
H(t)= \begin{cases}0, & \text { for } t<0, \\ 1, & \text { for } t>0 .\end{cases}
$$

The initial and boundary value problem (8) and (11)-(13) can be solved by applying the Laplace transform and the result is presented in Appendix A.

The radial velocity $u$ can be calculated by substituting Eq. (A6), (A7), or (A9) in Appendix A into Eq. (6). To determine the pressure disturbance $p$ in the leading order, we should linearize Eq. (7) and substitute Eq. (A6), (A7), or (A9) into the result. We thus obtain the near-field solutions for $u$ and $p$ as follows: (i) In region (I)

$$
u=0, \quad p=0 \text {. }
$$

(ii) In region (II)

$$
\begin{aligned}
u= & \epsilon A_{1} \Omega\left[J_{1}(\Omega r) \sin \left(\Omega t+\delta_{1}\right)\right. \\
& \left.-Y_{1}(\Omega r) \cos \left(\Omega t+\delta_{1}\right)\right]+\epsilon \Omega^{2} \int_{0}^{\infty} \frac{e^{-\zeta t}}{\zeta^{2}+\Omega^{2}} \\
& \times \frac{I_{1}(\zeta) K_{1}(\zeta r)-I_{1}(\zeta r) K_{1}(\zeta)}{\left[K_{1}(\zeta)\right]^{2}+\pi^{2}\left[I_{1}(\zeta)\right]^{2}} d \zeta, \\
p= & \gamma \epsilon A_{1} \Omega\left[J_{0}(\Omega r) \cos \left(\Omega t+\delta_{1}\right)\right. \\
& \left.+Y_{0}(\Omega r) \sin \left(\Omega t+\delta_{1}\right)\right] \\
& -\gamma \epsilon \Omega^{2} \int_{0}^{\infty} \frac{e^{-\zeta t}}{\zeta^{2}+\Omega^{2}} \\
& \times \frac{I_{1}(\zeta) K_{0}(\zeta r)+I_{0}(\zeta r) K_{1}(\zeta)}{\left[K_{1}(\zeta)\right]^{2}+\pi^{2}\left[I_{1}(\zeta)\right]^{2}} d \zeta,
\end{aligned}
$$

[ the definitions of $A_{1}$ and $\delta_{1}$ are given in Eq. (A8) in Appen$\operatorname{dix} A]$. (iii) In region (III)

$$
\begin{aligned}
u= & \epsilon \Omega^{2} \int_{0}^{\infty} \frac{e^{-\zeta(1-T)}-e^{-\zeta t}}{\zeta^{2}+\Omega^{2}} \\
& \times \frac{I_{1}(\xi r) K_{1}(\zeta)-I_{1}(\zeta) K_{1}(\zeta r)}{\left[K_{1}(\zeta)\right]^{2}+\pi^{2}\left[I_{1}(\zeta)\right]^{2}} d \zeta, \\
p= & \gamma \epsilon \Omega^{2} \int_{0}^{\infty} \frac{e^{-\zeta(t-T)}-e^{-\zeta t}}{\zeta^{2}+\Omega^{2}} \\
& \times \frac{I_{1}(\zeta) K_{0}(\zeta r)+I_{0}(\zeta r) K_{1}(\zeta)}{\left[K_{1}(\zeta)\right]^{2}+\pi^{2}\left[I_{1}(\zeta)\right]^{2}} d \zeta .
\end{aligned}
$$

That is, the expression of the solution in the near field is, in the $(r, t)$ plane, separated so as to describe the three regions: region (I) of constant state, region (II) pertaining to the body part of the wave, and region (III) pertaining to the tail. The boundaries between the three regions are the straight characteristic lines (see Fig. 1). The first terms on the righthand sides of Eqs. (15) and (16) correspond to the solutions for a periodic problem where the cylinder pulsates steadily (see, e.g., Ref. 4). The integral terms appearing in Eqs. (15)-(18) represent a transient effect owing to the fact that the cylinder begins the pulsation at $t=0$ and ceases it at $t=T$.

The profiles of the wave in the near field are depicted in Fig. 2 (a) and (b) by calculating Eqs. (15)-(18) numerically. It is clear from these figures that the radial velocity $u$ and the pressure disturbance $p$ are positive in the tail. The pressure disturbance on the surface of the cylinder, i.e., $p$ at $r=1$, is always positive for $t \geqslant T$, and it monotonically vanishes as $t \rightarrow \infty$ [see Eq. (18)], while $u$ at $r=1$ is zero for $t \geqslant T$ [see also Fig. 2(a) and (b) ]. Furthermore, the tail vanishes in the limit of $\Omega \rightarrow \infty$ for a fixed $M=\epsilon \Omega$ [see Eqs. (17) and (18) ]. These features are qualitatively unchanged from those of the spherical wave radiated by a sphere's sinusoidal pulsation for one period (see Ref. 5).

We shall derive an asymptotic representation for $\Phi$ in the tail as $t \rightarrow \infty$ at a fixed $r=O(1)$. Expanding the terms in curly brackets in Eq. (A9) in Appendix A in powers of $\xi$ and integrating the result term by term, we obtain

$$
\begin{aligned}
\Phi= & \frac{\epsilon T}{t^{2}}+\frac{\epsilon T^{2}}{t^{3}}+\frac{\epsilon T}{t^{4}}\left[\left(\frac{3}{2} r^{2}-3 \ln r\right)\right. \\
& \left.+T^{2}-\frac{6}{\Omega^{2}}+6 \ln 2 t-\frac{19}{2}\right] \\
& +O\left[(\ln t)^{2} t^{-5}\right] \quad \text { as } t \rightarrow \infty .
\end{aligned}
$$

In Refs. 8 and 9, however, it has been shown that $\Phi$ in the tail decays to zero in proportion to, in general, $t^{-1}$ as $t \rightarrow \infty$ at a fixed $r$ in the near field. The discrepancy is attributed to the boundary condition (12): because of the sinusoidal condition, the coefficient of $t^{-1}$ in Eq. (19) vanishes. Expansion

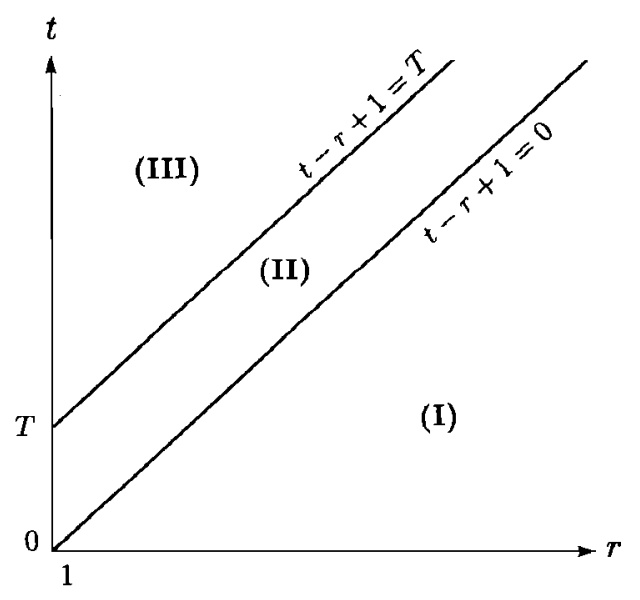

FIG. I. Three regions in the $(r, t)$ plane in the near field. 

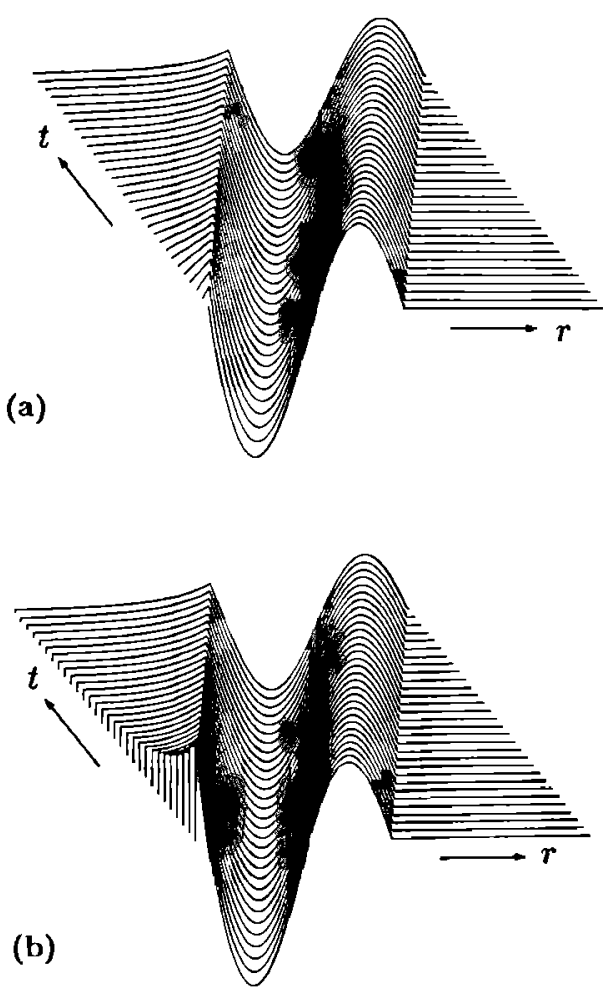

FIG. 2. The wave profiles from $t=T$ to $t=2 T(1 \leqslant r \leqslant 1+2 T)$ in the near field; $\epsilon=0.1, \Omega=0.8$, and $\gamma=1.4$. (a) The normalized velocity profiles, $u(r, t)$ (b) The profiles of the normalized pressure disturbance, $p(r, t)$.

(19) shows that the pressure disturbance in the tail decreases asymptotically in proportion to $t^{-3}$ and the velocity decreases in proportion to $t^{-4}$ as $t \rightarrow \infty$ at a fixed $r$ in the near field. On the other hand, in the case of the spherical wave, the tail decays exponentially in time at a fixed point. ${ }^{5}$

At large distances from the cylinder, we have the following asymptotic forms of $u$ and $p$ :

$$
u=\frac{\epsilon \Omega A}{\sqrt{r}} F(\varphi), \quad p=\gamma u,
$$

where

$$
\varphi \equiv \Omega(t-r+1), \quad A \equiv A_{1} \sqrt{(2 / \pi \Omega)},
$$

and the functional form of $F(\varphi)$ is defined as follows: (i) In region (I) $(\varphi<0)$

$$
F(\varphi)=0 \text {. }
$$

(ii) In region (II) $(0 \leqslant \varphi<2 \pi)$

$$
\begin{aligned}
F(\varphi)= & \sin (\varphi+\delta)-\frac{\Omega}{A \sqrt{2 \pi}} \int_{0}^{\infty} \frac{1}{\sqrt{\zeta}\left(\zeta^{2}+\Omega^{2}\right)} \\
& \times \frac{K_{1}(\zeta) e^{\zeta}}{\left[K_{1}(\zeta)\right]^{2}+\pi^{2}\left[I_{1}(\zeta)\right]^{2}} \exp \left(-\frac{\varphi}{\Omega} \zeta\right) d \zeta,
\end{aligned}
$$

where

$$
\delta \equiv \delta_{1}+\frac{3}{4} \pi-\Omega .
$$

(iii) In region (III) $(2 \pi \leqslant \varphi)$

$$
\begin{aligned}
F(\varphi)= & \frac{\Omega}{A \sqrt{2 \pi}} \\
& \times \int_{0}^{\infty}\left\{\frac{e^{\zeta T}-1}{\sqrt{\zeta}\left(\zeta^{2}+\Omega^{2}\right)} \frac{K_{1}(\zeta) e^{\zeta}}{\left[K_{1}(\zeta)\right]^{2}+\pi^{2}\left[I_{1}(\zeta)\right]^{2}}\right\} \\
& \times \exp \left(-\frac{\varphi}{\Omega} \zeta\right) d \zeta .
\end{aligned}
$$

Expressions (23) and (25) have been obtained by using the asymptotic expansions of the Bessel functions and modified Bessel functions for large arguments, Eqs. (B1)-(B4) in Appendix B. We shall remark that the function $F$ depends upon only one parameter $\Omega$. A typical profile of $F(\varphi)$ is presented in Fig. 3 for $\Omega=0.8$ by using numerical calculations.

The decay of the tail can be derived as

$$
F(\varphi)=B \varphi^{-5 / 2}+O\left(\varphi^{-7 / 2}\right) \quad \text { as } \varphi \rightarrow \infty \quad(r \gg 1) \text {. }
$$

where $B$ is a constant given by

$$
B=(3 \pi / 4 A) \sqrt{2 \Omega},
$$

which shows that the tail decays almost in proportion to $t^{-5 / 2}$ at a fixed large $r$ (a similar result has been derived by Whitham in the supersonic flow problem ${ }^{15}$ ). Equations (26) and (27) can be obtained by expanding the terms in curly brackets in Eq. (25) in powers of $\zeta$ and by integrating the result term by term.

Note that $F(\varphi)$ defined by Eqs. (23) and (25) satisfies an equation?

$$
\int_{0}^{\infty} F(\varphi) d \varphi=0 \text {. }
$$

The function $F$ of the present unsteady cylindrical waves is comparable to Whitham's $F$ function ${ }^{7}$ pertaining to the steady supersonic flow around an axisymmetrical body, because in both problems the wave behavior in the far field is determined by a mathematically equivalent far-field equation and such a function $F$.

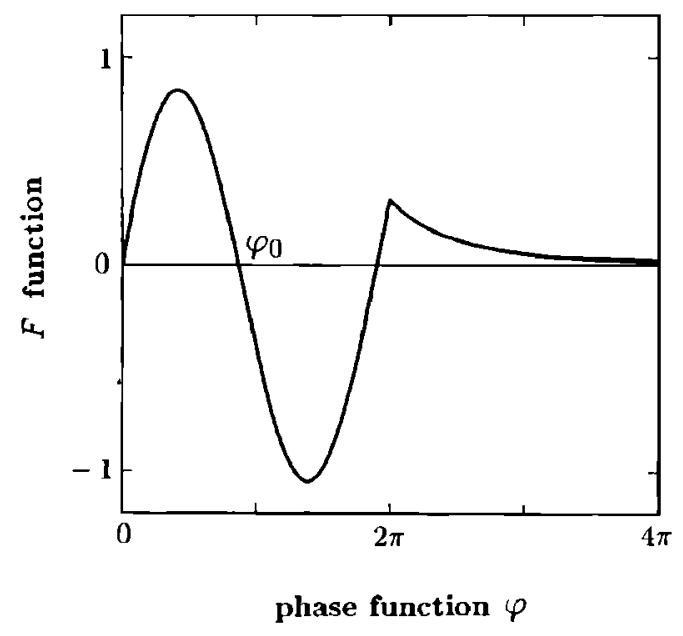

FIG. 3. Typical profile of the function $F$ for $\Omega=0.8$. 


\section{NONLINEAR PROPAGATION IN THE FAR FIELD}

\section{A. Exact solution of a far-field equation}

In the far field, the solution of the linear problem becomes invalid. As is well known, a far-field equation is given as $^{1,2}$

$$
\frac{\partial W}{\partial Z}-W \frac{\partial W}{\partial \varphi}=0
$$

where the dependent variable $W$ and the independent variable $\boldsymbol{Z}$ is defined as

$$
W=(u / \epsilon \Omega A) \sqrt{r}, \quad Z=\epsilon \Omega^{2} A(\gamma+1)(\sqrt{r}-1) .
$$

In Ref. 20, we have verified that a first-order far-field solution, in which the velocity $u$ is accurate up to $O(\epsilon / \sqrt{r})$, can be obtained by solving Eq. (29) under the boundary condition $^{21}$

$$
W=F(\varphi) \quad \text { at } Z=0,
$$

where the functional form of $F(\varphi)$ is given by Eq. (23) or Eq. (25), according as $0 \leqslant \varphi<2 \pi$ or $\varphi>2 \pi$.

Since Eq. (29) has the solution, in implicit form,

$$
W=W(\varphi+Z W) \text {, }
$$

the first-order far-field solution takes the form

$$
W=F(\hat{\varphi}), \quad \hat{\varphi}=\Omega(t-\eta+1),
$$

where $\eta$ is determined as a function of $r$ and $t$ by a relation

$$
\eta=r-\epsilon \Omega A(\gamma+1)(\sqrt{r}-1) F(\hat{\varphi}) .
$$

The new variable $\eta$ is the so-called strained coordinate. ${ }^{18}$ One may say that the effect of nonlinearity distorts the spatial coordinate in the phase function $\varphi$. The coordinate straining vanishes at $r=1$, i.e., $r=\eta=1$ there. It is obvious that the far-field solution, Eq. (33) with Eq. (34), matches with the asymptotic form of the near-field solution, Eq. (20). Accordingly, the profile of the wave in the far field also has no tail in the high-frequency limit of $\Omega \rightarrow \infty$ with $M$ being fixed.

Note that the strained coordinate in the body part of the wave $(0 \leqslant \hat{\varphi}<2 \pi)$ and that in the tail $(\hat{\varphi} \geqslant 2 \pi)$ are distinct, as in the problem for the spherical wave. ${ }^{s}$

The boundary between regions (I) and (II) is, at least until a shock wave is formed, defined by a straight characteristic line $t-r+1=0$, i.e.,

$$
\hat{\varphi}=\varphi=0 \text {. }
$$

The boundary between regions (II) and (III) is, however, not a straight line, although it is a characteristic curve,

$$
\begin{aligned}
t- & \left(r-(\gamma+1)(\sqrt{r}-1) \frac{\epsilon \Omega^{2}}{\sqrt{2 \pi}}\right. \\
& \left.\times \int_{0}^{\infty} \frac{1-e^{-T_{5}}}{\sqrt{\zeta}\left(\zeta^{2}+\Omega^{2}\right)} \frac{K_{1}(\zeta) e^{\zeta}}{\left[K_{1}(\zeta)\right]^{2}+\pi^{2}\left[I_{1}(\zeta)\right]^{2}} d \zeta\right) \\
& +1=T,
\end{aligned}
$$

i.e., $\hat{\varphi}=2 \pi$, until a shock crosses it (cf. Ref. 5 ).

\section{B. Formation of two shock waves}

The wave profile described by Eqs. (33) and (34) is distorted, as it propagates, due to the nonlinear effect. The nonlinear distortion eventually produces two shock waves. The shock formation distance $r_{s}$ can readily be derived from the condition ${ }^{18}$

$$
\frac{\partial r}{\partial \eta}=0
$$

Differentiating Eq. (34) with respect to $\eta$ and substituting Eq. (37) into the result yields

$$
1-Z \frac{d F(\hat{\varphi})}{d \hat{\varphi}}=0 .
$$

The normalized shock formation distance $Z_{s}$ is the smallest value of $Z$ satisfying Eq. (38). A shock wave is generated at $\varphi_{s}$, where $d F(\hat{\varphi}) / d \hat{\varphi}$ attains a positive maximum. Since the function $F$ defined by $\mathrm{Eq}$. (25) decreases monotonically with increase in its argument, no shock wave is produced in the tail. On the contrary, two shock waves can be formed in the body part of the wave, because $d F(\hat{\varphi}) / d \hat{\varphi}$ estimated by using Eq. (23) has two positive maxima in $0 \leqslant \hat{\varphi}<2 \pi$. The shock formation distance is thus given by

$Z_{s}=1 / G\left(\varphi_{s}\right)$ and $r_{s}=\left\{1+\left[Z_{s} / \epsilon \Omega^{2} A(\gamma+1)\right]\right\}^{2}$,

where the explicit form of $G(\hat{\varphi}) \equiv d F(\hat{\varphi}) / d \hat{\varphi}$ is

$$
\begin{aligned}
G(\hat{\varphi})= & \cos (\hat{\varphi}+\delta)+\frac{1}{A \sqrt{2 \pi}} \int_{0}^{\infty} \frac{\sqrt{\zeta}}{\zeta^{2}+\Omega^{2}} \\
& \times \frac{K_{1}(\zeta) e^{\zeta}}{\left[K_{1}(\zeta)\right]^{2}+\pi^{2}\left[I_{1}(\zeta)\right]^{2}} \exp \left(-\frac{\hat{\varphi}}{\Omega} \zeta\right) d \zeta .
\end{aligned}
$$

Clearly, one shock wave is generated at the boundary between regions (I) and (II), i.e., at $\hat{\varphi}=\varphi=0$; we shall call it the front shock. The shock formation distance is

$$
Z_{\mathrm{fs}}=1 / G(0) \text {, }
$$

where the subscript fs indicates the front shock.

Another shock, which is hereafter referred to as the rear shock, is generated at $\varphi_{\mathrm{rs}}$, where the subscript rs indicates the rear shock. Differentiating Eq. (40) with respect to $\hat{\varphi}$, we have the equations from which $\varphi_{\mathrm{rs}}$ is determined,

$$
\begin{aligned}
G^{\prime}\left(\varphi_{\mathrm{rs}}\right)= & -\sin \left(\varphi_{\mathrm{rs}}+\delta\right)-\frac{1}{A \Omega \sqrt{2 \pi}} \int_{0}^{\infty} \frac{\zeta \sqrt{\zeta}}{\zeta^{2}+\Omega^{2}} \\
& \times \frac{K_{1}(\zeta) e^{\zeta}}{\left[K_{1}(\zeta)\right]^{2}+\pi^{2}\left[I_{1}(\zeta)\right]^{2}} \\
& \times \exp \left(-\frac{\varphi_{\mathrm{rs}}}{\Omega} \zeta\right) d \zeta=0 \\
& \text { and } G^{\prime \prime}\left(\varphi_{\mathrm{rs}}\right)<0
\end{aligned}
$$

where the primes denote the differentiation with respect to $\hat{\varphi}$. The shock formation distance is

$$
Z_{\mathrm{rs}}=1 / G\left(\varphi_{\mathrm{rs}}\right) \text {. }
$$

We have aumerically confirmed that the rear shock is generated in the negative portion of the wave profile for an arbitrary $\Omega$, i.e., $W\left(\varphi_{\mathrm{rs}}\right)=F\left(\varphi_{\mathrm{rs}}\right)<0$ [see also Fig. 5(b)]. 
In the high-frequency limit of $\Omega \rightarrow \infty$,

$$
A \rightarrow 1, \quad \delta \rightarrow 0 \text {, }
$$

where Eqs. (B1) and (B2) in Appendix B have been used. Substituting Eq. (44) into Eqs. (40)-(43), we obtain

$$
Z_{\mathrm{fs}} \rightarrow 1, \quad \varphi_{\mathrm{rs}} \rightarrow 2 \pi, \quad Z_{\mathrm{rs}} \rightarrow 1 \quad \text { as } \Omega \rightarrow \infty .
$$

One can readily see from the second equation of Eqs. (39) and Eq. (45) that both of the front and rear shock formation distances approach the cylinder almost in proportion to $1 / \Omega$ as $\Omega \rightarrow \infty$ with the acoustic Mach number $M=\epsilon \Omega$ being fixed. By detailed numerical calculations, we have found that $Z_{\mathrm{fs}}<Z_{\mathrm{rs}}<1$ for $\Omega<\Omega_{c}$ and that $Z_{\mathrm{rs}}<\boldsymbol{Z}_{\mathrm{fs}}<1$ for $\Omega_{c}<\Omega$, where $\Omega_{c}=17.586$. This dependence of the shock formation distances on $\Omega$ is very similar to that of the spherical wave in Ref. 5. In Fig. 4, $1-Z_{\mathrm{rs}}$ and $1-Z_{\mathrm{rs}}$ are depicted for varying $\Omega$ by using numerical calculations, where the reversion noted above is evidently shown. Figure 4 also shows that $Z_{\mathrm{fs}}$ monotonically approaches to unity with increase in $\Omega$, while $Z_{\mathrm{rs}}$ has a minimum value at $\Omega=1.324$.

\section{PROPAGATION OF AN N WAVE WITH A TAIL}

Since we are concerned with the case of sufficiently large $R_{e}$, the shock waves can be regarded as discontinuities. The propagation of the weakly nonlinear waves accompanied by such shocks can be analyzed with the help of the equal-areas rule. ${ }^{6.7}$ A shock is hereby located in the phase so as to cut off equal areas of the triple-valued waveform satisfying the farfield equation (29). The formula can be written as ${ }^{7}$

$$
\begin{aligned}
& \varphi_{s}=\hat{\varphi}_{1}-Z W\left(\hat{\varphi}_{1}\right)=\hat{\varphi}_{2}-Z W\left(\hat{\varphi}_{2}\right), \\
& \frac{Z}{2}\left\{\left[W\left(\hat{\varphi}_{2}\right)\right]^{2}-\left[W\left(\hat{\varphi}_{1}\right)\right]^{2}\right\}=\int_{\hat{\varphi}_{1}}^{\hat{\varphi}_{2}} W(\hat{\varphi}) d \hat{\varphi},
\end{aligned}
$$

where a discontinuity is supposed to exist at $\varphi=\varphi_{s}$, and subscripts 1 and 2 indicate the values of $\hat{\varphi}$ immediately in front of and behind the discontinuity, respectively.

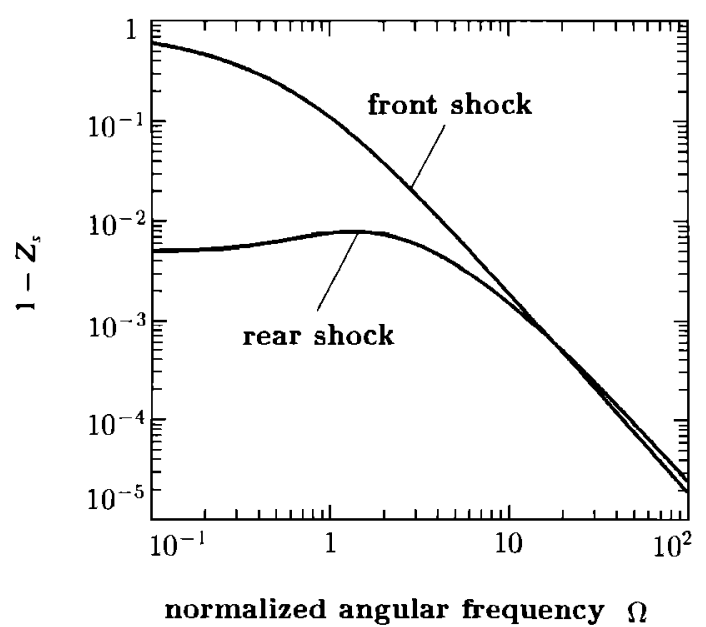

FIG. 4. The difference between the normalized shock formation distance $Z$ 、 and its limiting value 1 .

\section{A. Evolution of the front shock}

For the front shock, it can be seen that, as $Z\left(\geqslant Z_{\mathrm{fs}}\right)$ increases, $\hat{\varphi}_{1}(\leqslant 0)$ decreases monotonically and $\hat{\varphi}_{2}$ approaches to $\varphi_{0}$, where $\varphi_{0}$ is a constant depending upon $\Omega$ defined by

$$
F\left(\varphi_{0}\right)=0 \quad\left(0<\varphi_{0}<\pi\right),
$$

(see Fig. 3). The system (46) and (47) can therefore be reduced to

$$
\begin{aligned}
& \varphi_{s}=\hat{\varphi}_{1}=\hat{\varphi}_{2}-Z F\left(\hat{\varphi}_{2}\right), \\
& \frac{Z}{2}\left[F\left(\hat{\varphi}_{2}\right)\right]^{2}=\int_{0}^{\hat{\varphi}_{2}} F(\hat{\varphi}) d \hat{\varphi},
\end{aligned}
$$

since $W(\hat{\varphi})=F(\hat{\varphi})$ and $F(\hat{\varphi})=0$ for $\hat{\varphi}_{1} \leqslant 0$. With the help of the explicit form of $F$, Eq. (23), we can solve Eq. (50) to obtain $\hat{\varphi}_{2}$ as a function of $Z$. Substituting the result into Eq. (49) gives $\varphi_{\mathrm{s}}$ for any $Z$ ( $\geqslant Z_{\mathrm{fs}}$ ).

As $Z \rightarrow \infty$, we have $\hat{\varphi}_{2} \rightarrow \varphi_{0}$, and hence we have $W\left(\hat{\varphi}_{2}\right) \sim G\left(\varphi_{0}\right)\left(\hat{\varphi}_{2}-\varphi_{0}\right)$ by means of the relations $W(\hat{\varphi})=F(\hat{\varphi})$ and $G(\hat{\varphi})=d F(\hat{\varphi}) / d \hat{\varphi}$ [see also Eq. $(40)]$. Replacing $\hat{\varphi}_{2}$ on the right-hand side of Eq. (50) by $\varphi_{0}$ gives an asymptotic representation for $W\left(\hat{\varphi}_{2}\right)$ as

$$
W\left(\hat{\varphi}_{2}\right)=F\left(\hat{\varphi}_{2}\right) \sim \sqrt{(2 S / Z)},
$$

where $S$ is a constant depending upon $\Omega$ defined by

$$
S=\int_{0}^{4} F(\hat{\varphi}) d \hat{\varphi}
$$

Substituting Eq. (51) into the right-hand side of Eq. (49), we obtain an asymptotic formula of the location of the front shock,

$$
\varphi_{s} \sim \widehat{\varphi}_{2}-\sqrt{2 S Z} \sim \varphi_{0}-\sqrt{2 S Z} .
$$

Equations (51)-(53) are the well-known results (see, e.g., Ref. 7).

\section{B. Evolution of the rear shock and the tail}

The rear shock, which emerges in region (II), crosses the boundary between regions (II) and (III) in an initial stage of its evolution. To determine the location of the rear shock by means of the equal-areas rule, one should replace $\varphi$ in Eq. (23) by $\hat{\varphi}_{1}$ and substitute the result into $W\left(\hat{\varphi}_{1}\right)$ in Eqs. (46) and (47). For $W\left(\hat{\varphi}_{2}\right)$ in Eqs. (46) and (47), one should use Eq. (23) or Eq. (25), according as $\hat{\varphi}_{2}<2 \pi$ or $\hat{\varphi}_{2} \geqslant 2 \pi$. The system (46) and (47) thus determines $\hat{\varphi}_{1}$ and $\hat{\varphi}_{2}$ for any $Z\left(\geqslant Z_{\mathrm{rs}}\right)$, so that we can obtain the location of the rear shock.

As $Z \rightarrow \infty$, it can readily be seen that $\hat{\varphi}_{1}$ approaches $\varphi_{0}$ (see Fig. 3) and that $\hat{\varphi}_{2}$ monotonically increases to infinity. We therefore have $W\left(\hat{\varphi}_{1}\right) \sim G\left(\varphi_{0}\right)\left(\hat{\varphi}_{1}-\varphi_{0}\right)$ and

$$
W\left(\hat{\varphi}_{2}\right) \sim B \hat{\varphi}_{2}^{-5 / 2} \ll 1,
$$

where Eq. (26) has been used. The presence of the tail is reflected in Eq. (54). Clearly, in an ordinary balanced $N$ wave, $W\left(\hat{\varphi}_{2}\right)=0$. Substituting Eq. (54) into Eq. (47) and replacing $\hat{\varphi}_{1}$ on the right-hand side of Eq. (47) by $\varphi_{0}$ yields

$$
W\left(\hat{\varphi}_{1}\right) \sim-\sqrt{(2 S / Z)} .
$$

Here we have used Eq. $(28)$ and the fact that $W\left(\hat{\varphi}_{1}\right)<0$. From Eqs. (46) and (55), we can derive 


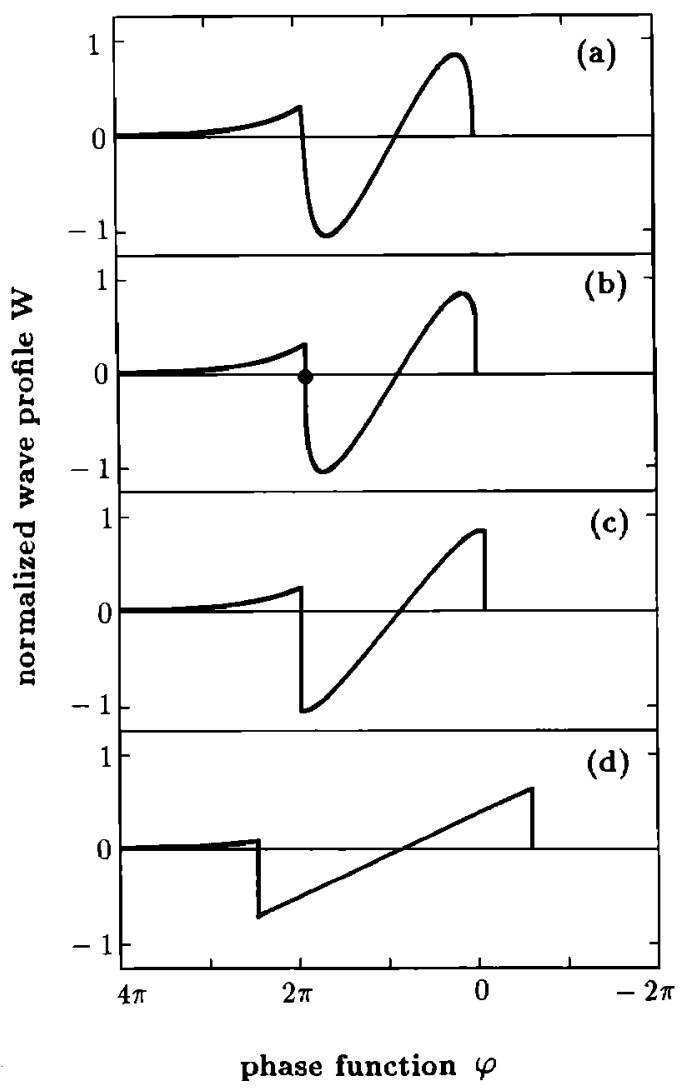

FIG. 5. The nonlinear distortion of the waveform and formation of an $N$ wave with a tail. The figures are depicted by using the far-field solution, Eqs. (33) and (34), with the help of the equal-areas rule for $\epsilon=0.1, \Omega=0.8$, and $\gamma=1.4$. (a) At the moment of formation of the front shock, $t=55.4$; $Z_{\mathrm{is}}=0.853$. (b) At the moment of formation of the rear shock, $t=80.0$; $\mathcal{Z}_{\mathrm{rs}}=0.993$. A black circle indicates the shock formation point; $W=F\left(\varphi_{\mathrm{rs}}\right)=-0.032, \varphi_{\mathrm{rs}}=5.934$. (c) At the moment when both of the two shocks attain the almost maximum strengths, $t=195$. (d) The profile of $W$ grows into an $N$ wave with a tail; $t=2188$.

$$
\varphi_{s} \sim \hat{\varphi}_{2} \sim \hat{\varphi}_{1}+\sqrt{2 S Z} \sim \varphi_{0}+\sqrt{2 S Z} .
$$

Note that Eqs. (55) and (56) are the same results as in the case of the balanced $N$ wave with no tail (see Ref. 7). It follows from Eqs. (54), (56), and (30) that the radial veloc-

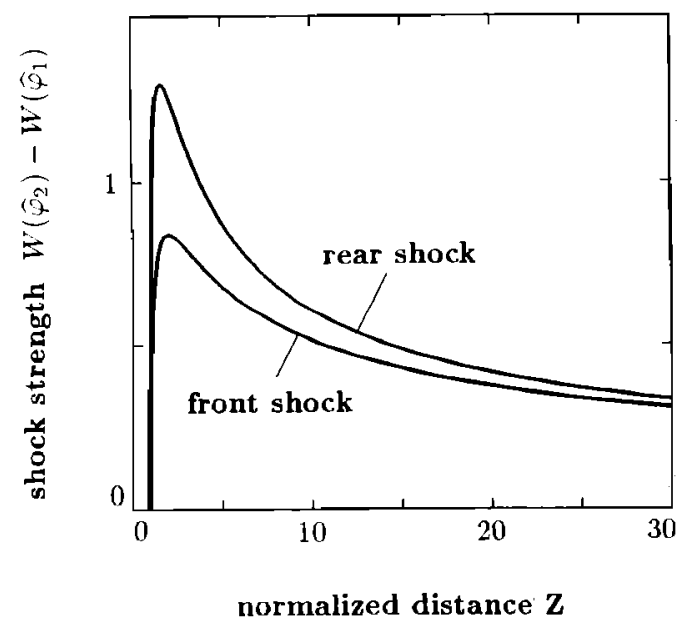

FIG. 6. The growth and attenuation of shock strength, $\boldsymbol{W}\left(\hat{\varphi}_{2}(\mathcal{Z})\right)-\boldsymbol{W}\left(\hat{\varphi}_{1}(\mathcal{Z})\right) ; \Omega=0.8$.

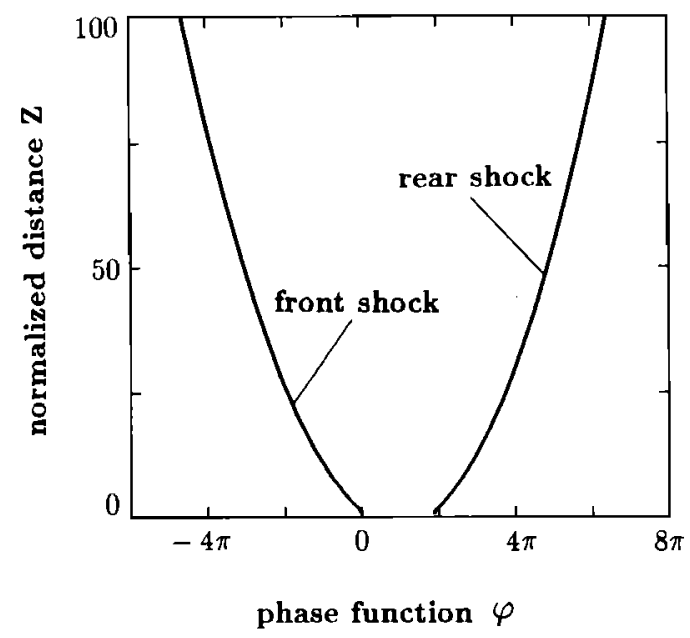

FIG. 7. The paths of the two shocks in the $(\varphi, Z)$ plane; $\Omega=0.8$.

ity $u$ immediately behind the rear shock decays as $r^{-9 / 8}$ as the wave propagates; a similar result has been obtained in the supersonic flow problem. ${ }^{15}$

Consequently, the whole profile at large distances from the cylinder is an $N$ wave with a tail,

$W(\varphi, Z)$

$$
= \begin{cases}0, & \text { for } \varphi<\varphi_{0}-\sqrt{2 S Z}, \\ \frac{\varphi_{0}-\varphi}{Z}, & \text { for } \varphi_{0}-\sqrt{2 S Z} \leqslant \varphi<\varphi_{0}+\sqrt{2 S Z}, \\ B \varphi^{-5 / 2}, & \text { for } \varphi_{0}+\sqrt{2 S Z} \leqslant \varphi .\end{cases}
$$

The tail immediately behind the rear shock decays as $Z^{-5 / 4}$ as the wave propagates, whereas the body part immediately in front of the rear shock decays as $Z^{-1 / 2}$. The whole profile accordingly approaches to the balanced $N$ wave with no tail in the limit of $Z \rightarrow \infty$. We shall remark that the decay rate of the tail is very low compared with that of the spherical wave treated in Ref. 5, where the tail immediately behind the rear shock decays as $e^{-\sqrt{z}}$ as the wave propagates.

The numerical results of the analysis by making use of the equal-areas rule are shown in Figs. 5-7. Figure 5(a) and (b) give the wave profiles at the moment of the front and rear shock formation, respectively. At first each shock grows and attains the maximum strength; thereafter its strength diminishes. Figure 5 (c) shows the wave profile of $W$ at the moment when both of the two shocks attain the almost maximum strengths. The profile of a typical $N$ wave with the tail is given in Fig. 5(d).

In Fig. 6, the shock strength, $W\left(\hat{\varphi}_{2}\right)-W\left(\hat{\varphi}_{1}\right)$, is shown as a function of $Z$. Owing to the presence of the positive tail, the rear shock is stronger than the front one at any $Z$ [see Eq. (28)].

The shock path in the $(\varphi, Z)$ plane, i.e., $\varphi_{s}$ as a function of $Z$, is presented in Fig. 7; the asymptote is a parabola. 


\section{APPENDIX A: NEAR-FIELD SOLUTION FOR THE VELOCITY POTENTIAL}

Applying the Laplace transform to the wave equation (11) and using the initial conditions (8) gives

$$
\frac{\partial^{2} \Psi}{\partial r^{2}}+\frac{1}{r} \frac{\partial \Psi}{\partial r}-s^{2} \Psi=0
$$

where

$$
\Psi(r, s) \equiv \int_{0}^{\infty} e^{-s t} \Phi(r, t) d t
$$

The boundary condition (12) can also be transformed into

$$
\frac{\partial \Psi}{\partial r}=\frac{\epsilon \Omega^{2}}{s^{2}+\Omega^{2}}\left(1-e^{-s T}\right) \quad \text { at } r=1 .
$$

Solving Eq. (A1) under the boundary condition (A3) and the radiation condition at infinity, we have

$$
\Psi(r, s)=\frac{\epsilon \Omega^{2}}{s\left(s^{2}+\Omega^{2}\right)} \frac{K_{0}(s r)}{K_{1}(s)}\left(e^{-s T}-1\right),
$$

where $K_{n}$ denotes the modified Bessel function of the second kind of order $n$ (see Ref. 22 for the definition). The velocity potential $\Phi$ can then be given by the inverse transformation as

$$
\begin{aligned}
\Phi(r, t)= & \frac{1}{2 \pi i} \int_{\sigma-i \infty}^{\sigma+i \infty} \frac{\epsilon \Omega^{2}}{s\left(s^{2}+\Omega^{2}\right)} \frac{K_{0}(s r)}{K_{1}(s)} \\
& \times\left(e^{s(t-T)}-e^{s t}\right) d s,
\end{aligned}
$$

where $\sigma$ is a positive number such that the integral in Eq. (A2) absolutely converges for all $s$ with $\operatorname{Re}[s]>\sigma$. We can reduce the representation (A5) to the form more tractable for our purpose (see Appendix C): (i) In region (I) $(t-r+1<0)$

$$
\Phi=0 \text {. }
$$

(ii) In region (II) $(0 \leqslant t-r+1<T)$

$$
\begin{aligned}
\Phi= & \epsilon A_{1}\left[Y_{0}(\Omega r) \cos \left(\Omega t+\delta_{1}\right)-J_{0}(\Omega r) \sin \left(\Omega t+\delta_{1}\right)\right] \\
& -\epsilon \Omega^{2} \int_{0}^{\infty} \frac{e^{-\zeta t}}{\zeta\left(\zeta^{2}+\Omega^{2}\right)} \\
& \times \frac{I_{1}(\zeta) K_{0}(\zeta r)+I_{0}(\zeta r) K_{1}(\zeta)}{\left[K_{1}(\zeta)\right]^{2}+\pi^{2}\left[I_{1}(\zeta)\right]^{2}} d \zeta
\end{aligned}
$$

Here, $J_{n}$ and $Y_{n}$ denote the Bessel functions of the first and the second kind of order $n$, respectively, and $I_{n}$ is the modified Bessel function of the first kind of order $n$, and constants $A_{1}$ and $\delta_{1}$ are defined by

$$
\begin{aligned}
& A_{1}=\left\{\left[J_{1}(\Omega)\right]^{2}+\left[Y_{1}(\Omega)\right]^{2}\right\}^{-1 / 2}, \\
& \delta_{1}=\tan ^{-1}\left[Y_{1}(\Omega) / J_{1}(\Omega)\right]
\end{aligned}
$$

(see Ref. 22 for the definitions of $J_{n}, Y_{n}$, and $I_{n}$ ). (iii) In region (III) $(T \leqslant t-r+1)$

$$
\begin{aligned}
\Phi= & \epsilon \Omega^{2} \int_{0}^{\infty}\left\{\frac{e^{\zeta T}-1}{\xi\left(\xi^{2}+\Omega^{2}\right)}\right. \\
& \left.\times \frac{I_{1}(\zeta) K_{0}(\zeta r)+I_{0}(\xi r) K_{1}(\zeta)}{\left[K_{1}(\xi)\right]^{2}+\pi^{2}\left[I_{1}(\xi)\right]^{2}}\right\} e^{-\zeta t} d \zeta .
\end{aligned}
$$

\section{APPENDIX B: FORMULAS RELATED TO THE BESSEL FUNCTIONS AND THE MODIFIED BESSEL FUNCTIONS}

Formulas related to the Bessel functions and the modified Bessel functions are listed below (see Ref. 22), which are used in Secs. II and III, and Appendix C. In this Appendix, $n$ is an integer and $z$ is a complex number.

The asymptotic expansions for large arguments are given as

$$
\begin{aligned}
& J_{n}(z) \sim \sqrt{\frac{2}{\pi z}} \cos \left(z-\frac{n \pi}{2}-\frac{\pi}{4}\right) \quad(z \rightarrow \infty), \\
& Y_{n}(z) \sim \sqrt{\frac{2}{\pi z}} \sin \left(z-\frac{n \pi}{2}-\frac{\pi}{4}\right) \quad(z \rightarrow \infty), \\
& I_{n}(z) \sim \frac{1}{\sqrt{2 \pi z}} e^{z} \quad\left(|z| \rightarrow \infty,|\arg z|<\frac{\pi}{2}\right), \\
& K_{n}(z) \sim \sqrt{\frac{\pi}{2 z}} e^{-z} \quad\left(|z| \rightarrow \infty,|\arg z|<\frac{3}{2} \pi\right) .
\end{aligned}
$$

The modified Bessel functions of the second kind are connected to the Bessel functions by the relations $K_{n}(z)$

$$
=\left\{\begin{array}{l}
\frac{1}{2} \pi i e^{n \pi i / 2} H_{n}^{(1)}\left(z e^{\pi i / 2}\right) \quad(-\pi<\arg z \leqslant \pi / 2), \\
-\frac{1}{2} \pi i e^{-n \pi i / 2} H_{n}^{(2)}\left(z e^{-\pi i / 2}\right) \quad(-\pi / 2<\arg z \leqslant \pi),
\end{array}\right.
$$

where

$H_{n}^{(1)}(z)=J_{n}(z)+i Y_{n}(z), \quad H_{n}^{(2)}(z)=J_{n}(z)-i Y_{n}(z)$.

The following relation also holds for the modified Bessel functions:

$$
K_{n}\left(z e^{i n \pi i}\right)=K_{n}(z) e^{-n m \pi i}-\pi i \lim _{v \rightarrow n} \frac{\sin v m \pi}{\sin v \pi} I_{v}(z),
$$

where $m$ is an integer.

\section{APPENDIX C: EVALUATION OF THE INVERSION INTEGRAL}

The inversion integral

$$
\Phi(r, t)=\frac{1}{2 \pi i} \int_{\sigma-i \infty}^{\sigma+i \infty} e^{s t} \Psi(r, s) d s,
$$

signifies that the integration is to be performed along a straight line $s=\sigma$ from $\sigma-i_{\infty}$ to $\sigma+i_{\infty}$, in a complex number plane. Here, $\sigma$ is a positive constant such that the integral in Eq. (A2) in Appendix A absolutely converges for all $s$ with $\operatorname{Re}[s]>\sigma$. In the following, we shall evaluate the integral in Eq. (C1) in terms of a contour integral in the $s$ plane.

The function $\Psi(r, s)$ given by Eq. (A4) in Appendix A can be rewritten into

$$
\Psi(r, s)=\epsilon \Omega^{2}\left[\Psi_{1}(r, s)+\Psi_{2}(r, s)\right],
$$

where 


$$
\begin{aligned}
& \Psi_{1}(r, s)=\frac{1}{s\left(s^{2}+\Omega^{2}\right)} \frac{K_{0}(s r)}{K_{1}(s)} e^{-s T}, \\
& \Psi_{2}(r, s)=-\frac{1}{s\left(s^{2}+\Omega^{2}\right)} \frac{K_{0}(s r)}{K_{1}(s)} .
\end{aligned}
$$

Clearly, both the functions $\Psi_{1}$ and $\Psi_{2}$ have simple poles at $s= \pm i \Omega$. The function $\left(e^{-s T}-1\right) / s\left(s^{2}+\Omega^{2}\right)$ however is analytic at $s= \pm i \Omega$ since $T=2 \pi / \Omega$. Both $K_{0}(s r)$ and $K_{1}(s)$, each of which has a branch point at the origin, have no zeros and no poles in a region $-\pi \leqslant \arg s \leqslant \pi$ and $s \neq 0$ (see the book by Watson ${ }^{23}$ ). Accordingly, $\Psi$ is single-valued and analytic in the region. We also need to examine an asymptotic behavior of $e^{s t} \Psi(r, s)$ as $|s| \rightarrow \infty$. Using Eq. (B4) in Appendix B, we have

$$
\begin{aligned}
& e^{s t} \Psi_{1}(r, s) \sim \frac{e^{(t-r+1-T) s}}{s\left(s^{2}+\Omega^{2}\right) \sqrt{r}}, \\
& e^{s t} \Psi_{2}(r, s) \sim-\frac{e^{(t-r+1) s}}{s\left(s^{2}+\Omega^{2}\right) \sqrt{r}},
\end{aligned}
$$

as $|s| \rightarrow \infty$ and $|\arg s|<\frac{3}{2} \pi$.

We now evaluate the inversion integral (C1) in the following way: (i) In the case of $t-r+1<0$, we shall consider

$$
\int_{\Gamma_{1}} e^{s t}\left[\Psi_{1}(r, s)+\Psi_{2}(r, s)\right] d s,
$$

taken along the contour $\Gamma_{1}$ shown in Fig. $\mathrm{Cl}$, consisting of a semicircle of radius $R$ and the straight line joining its end points. Since there are no poles of $e^{s t} \Psi(r, s)$ inside $\Gamma_{1}$, the contour integral is zero by Cauchy's theorem. In the limit of $R \rightarrow \infty$, it follows from Eq. (C4) that the integral along the semicircle tends to zero, and hence we have

$$
\Phi(r, t)=0 \text {. }
$$

(ii) In the case of $0 \leqslant t-r+1<T$, we shall consider the sum of the two contour integrals,

$$
\int_{\Gamma_{1}} e^{s t} \Psi_{1}(r, s) d s+\int_{\Gamma_{2}} e^{s t} \Psi_{2}(r, s) d s
$$

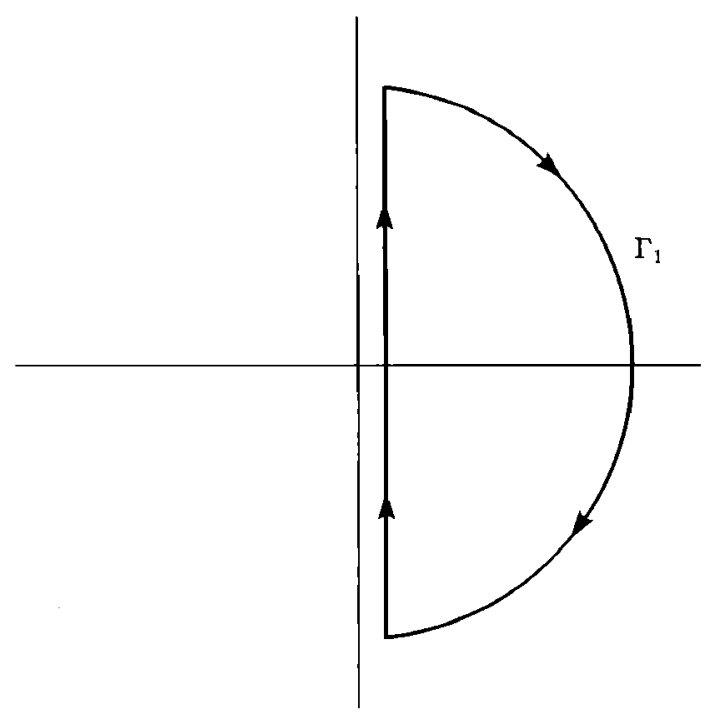

FIG. C1. The contour $\Gamma_{1}$ in the $s$ plane.

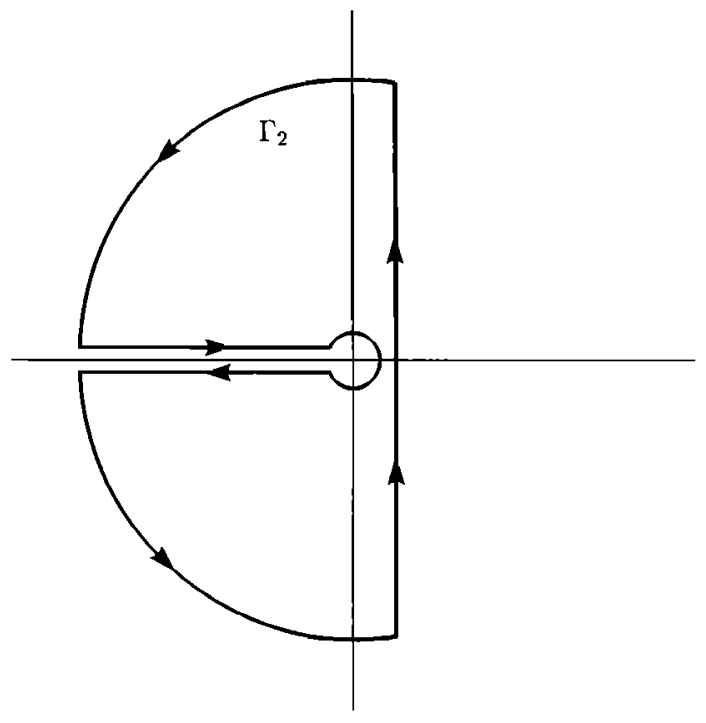

FIG. C2. The contour $\Gamma_{2}$ in the s plane.

Here the contour $\Gamma_{2}$ is shown in Fig. C2, which is chosen so that it encloses no singularities except for simple poles $s= \pm i \Omega$ and that the integrand becomes single-valued within and on the contour $\Gamma_{2}$. In the same manner as in the case (i), the first integral in Eq. (C7) is zero. The second integral can be evaluated with the help of the theorem of residues. Using the series expansions, ${ }^{22}$ we have

$$
K_{0}(s r) \rightarrow-\ln s, \quad K_{1}(s) \rightarrow 1 / s \quad \text { as } s \rightarrow 0 .
$$

The integral along a small circle of $\Gamma_{2}$ therefore vanishes as its radius tends to zero. The integral along a large circle also vanishes as its radius tends to infinity [see the second of Eq. (C4) ]. Consequently $\Phi$ is given by the sum of the residues and the integrals along the semi-infinite lines $s=\zeta e^{ \pm \pi i}(\zeta$ is a positive real number), i.e.,

$$
\begin{aligned}
\Phi(r, t)= & \frac{\epsilon}{2}\left(e^{i \Omega t} \frac{K_{0}\left(\Omega r e^{\pi / 2}\right)}{K_{1}\left(\Omega e^{\pi i / 2}\right)}+e^{-i \Omega t} \frac{K_{0}\left(\Omega r e^{-\pi i / 2}\right)}{K_{1}\left(\Omega e^{-\pi i / 2}\right)}\right) \\
& -\frac{1}{2 \pi i} \int_{0}^{\infty} \frac{\epsilon \Omega^{2}}{\zeta\left(\zeta^{2}+\Omega^{2}\right)} \\
& \times\left(\frac{K_{0}\left(\zeta r e^{\pi i}\right)}{K_{1}\left(\zeta e^{\pi i}\right)}-\frac{K_{0}\left(\zeta r e^{-\pi i}\right)}{K_{1}\left(\zeta e^{-\pi i}\right)}\right) e^{-\zeta t} d \zeta .
\end{aligned}
$$

Using formulas (B5)-(B7) in Appendix B, we can transform Eq. (C9) to Eq. (A7) in Appendix A. (iii) In the case of $T \leqslant t-r+1$, we shall consider

$$
\int_{\Gamma_{2}} e^{s /} \Psi(r, s) d s
$$

Since there are no poles of $e^{s t} \Psi(r, s)$ inside $\Gamma_{2}$, the contour integral is zero by Cauchy's theorem. We thus obtain Eq. (A9) in Appendix A.

\footnotetext{
' K. A. Naugol'nykh, S. I. Soluyan, and R. V. Khokhlov, "Cylindrical waves of finite amplitude in a dissipative medium," Vestnik, Moscow State Univ., Phys. Astron., ser. 34, 65-71 (1962).

${ }^{2}$ D. T. Blackstock, "On plane, spherical, and cylindrical sound waves of
} 
finite amplitude in lossless fluids," J. Acoust. Soc. Am. 36, 217-219 (1964).

${ }^{3}$ P. L. Sachder and A. R. Seebass, "Propagation of spherical and cylindrical $N$-waves," J. Fluid. Mech. 58, 197-205 (1973).

${ }^{4}$ J. H. Ginsberg, "Propagation of nonlinear acoustic waves induced by a vibrating cylinder. I. The two-dimensional case," J. Acoust. Soc. Am. 64, 1671-1678 (1978)

${ }^{5}$ Y. Inoue, T. Yano, and H. Tsujimura, "Propagation of acoustic waves and shock waves radiated by a sinusoidal pulsation of a sphere during a single period," Fluid Dyn. Res. 4, 215-227 (1988).

${ }^{\circ}$ L. D. Landau and E. M. Lifshitz, Fluid Mechanics (Pergamon, Oxford, 1987), 2nd ed., Sec. 102. Note that, in Fig. 84 in Sec. 102, the tail of the wave has negative fluid velocity, while in our result it has positive fluid velocity [ see Fig. 2(a) ]. The discrepancy is caused by the difference of the boundary conditions. In fact, if the wave radiated by a cylinder (or sphere) which expands for only a half-cycle of a sinusoid is considered, the tail of the wave profile has a negative fluid velocity.

${ }^{7}$ G. B. Whitham, Linear and Nonlinear Waves (Wiley, New York, 1974), Sec. 9.3.

${ }^{8}$ See Ref. 6, Sec. 71.

${ }^{9}$ See Ref. 7, Sec. 7.4.

${ }^{10}$ Supposing that an acoustic Mach number $M=\epsilon \Omega$ is sufficiently small and that an acoustic Reynolds number is sufficiently large, Inoue and Gotoh have classified the structures of sound field radiated by a uniformly pulsating sphere into the following three types: (a) $\epsilon \ll<1$ and $\Omega \gg 1$; (b) $\epsilon \ll 1$ and $\Omega=O(1) ;(c) \epsilon=O(1)$ and $\Omega \ll 1$. See Y. Inoue and $Y$. Gotoh, "Formation of weak shock waves caused by a sphere pulsating at large amplitude and low frequency," J. Sound Vib. 146, 269-280 (1991). In the present paper, we are chiefly concerned with the case (b).

" Here, $\Omega / 2 \pi$ also denotes the ratio of the mean radius $\bar{R}$ to a typical wavelength of the radiated sound, $2 \pi c / \omega$.

${ }^{12} \mathrm{O}$. V. Rudenko and S. I. Soluyan, Theoretical Foundations of Nonlinear Acoustics (Consultant Bureau, New York, 1977), p. 11.

". D. G. Crighton and J. F. Scott, "Asymptotic analysis of model equations in nonlinear acoustics," Philos. Trans. R. Soc. London Ser. A 292, 101134 (1979)
${ }^{14} \mathrm{Y}$. Inoue and T. Okigami, "Propagation of finite amplitude sound waves radiated from an oscillating rigid sphere," J. Sound Vib. 118, 199-207 (1987).

15 G. B. Whitham, "The behavior of supersonic flow past a body of revolution, far from the axis," Proc. R. Soc. London Ser. A 201, 89-109 (1950). ${ }^{16}$ See Ref. 10.

${ }^{17}$ Note that a large-amplitude sinusoidal surface displacement of an acoustically compact sphere produces a nonsinusoidal volume outflow in the neighborhood of the sphere. The outflow generates the acoustic waves composed of the fundamental and two harmonics in the leading order. This leads to the two-shocks phenomenon in the far field, if the ratio of the surface displacement to the mean radius of the sphere is larger than $1 / 7$; otherwise only one shock is formed in each one wave cycle. See Ref. 10.

${ }^{18} \mathrm{Y}$. Inoue, S. Ishii, and T. Okigami, "Propagation of finite amplitude sound waves radiated from a pulsating sphere," J. Sound Vib. 106, 257265 (1986).

${ }^{19}$ Replacing $\gamma-1$ in Eq. (4) by the nonlinearity parameter $B / A$, one can apply the present analysis to other fluids than an ideal gas. Here $B / A$ is determined from an assumed pressure-density relation. See, for example, R. T. Beyer, "Parameter of nonlinearity in fuids," J. Acoust. Soc. Am. 32, 719-721 (1960).

${ }^{20} \mathrm{~T}$. Yano and Y. Inoue, "Weakly nonlinear waves generated by vibration of a spherical body," J. Acoust. Soc. Am. 91, 69-78 (1992).

${ }^{21}$ The variable $Z$ is introduced to describe the far-field behavior of the wave. Accordingly, $Z$ is a long space scale compared with the characteristic length in the near field, i.e., a typical wavelength. The limit $Z \rightarrow 0$ in the far field therefore corresponds to the limit $r \rightarrow \infty$ in the near field, as in the usual method of matched asymptotic expansions. The main finite amplitude effect (nonlinear waveform distortion and shock formation) occurs in a region $0<Z \leqslant Z \approx 1$ ( $Z$, is $Z$ evaluated at a shock formation distance), where Eq. (29) is applicable. See Ref. 20 for details.

${ }^{22}$ Handbook of Mathematical Functions, edited by M. Abramowitz and I. A. Stegun (Dover, New York, 1970), Chap. 9.

${ }^{23}$ G. N. Watson, A Treatise on the Theory of Bessel Functions (Cambridge U.P., New York, 1966), pp. 511-513. 